\title{
Changes to the bladder epithelial barrier are associated with ketamine-induced cystitis
}

\author{
QIXIN DUAN ${ }^{1}$, TIANPENG WU ${ }^{2}$, XIAOCHUN YI ${ }^{3}$, LINGQI LIU $^{2}$, JIANTAO YAN $^{3}$ and ZHONGJIE LU ${ }^{4}$ \\ Departments of ${ }^{1}$ Critical Medical Care and ${ }^{2}$ Urology, Renmin Hospital of Wuhan University, Wuhan, Hubei 430060; \\ ${ }^{3}$ Department of Urology and Transplantation, The First People's Hospital of Yueyang, Yueyang, Hunan 414000; \\ ${ }^{4}$ Department of Urology, Renmin Hospital of Hanchuan, Hanchuan, Hubei 432100, P.R. China
}

Received December 30, 2015; Accepted January 20, 2017

DOI: 10.3892/etm.2017.4913

\begin{abstract}
The aim of the present study was to investigate the changes of the bladder epithelial barrier in the pathogenesis of ketamine-induced cystitis (KIC). A total of 60 female mice were randomly allocated into control and ketamine groups, which received daily intraperitoneal injections of saline and ketamine, respectively. Micturition behavior was recorded in 2-h intervals at the end of 4, 8 and 12 weeks, and bladders were harvested for subsequent analyses. Routine hematoxylin and eosin staining was performed on the bladders and histopathological changes were analyzed using light microscopy. The distribution of zonula occludens-1 (ZO-1) protein was determined by immunohistochemical analysis. The ultrastructure of umbrella cells was observed using a transmission electron microscope (TEM). Ketamine-addicted mice exhibited a significantly increased frequency of micturitions following 8 and 12 weeks of ketamine treatment $(\mathrm{P}<0.05$ and $\mathrm{P}<0.01$, respectively). Suburothelial congestion and infiltration of mononuclear cells was observed in ketamine-addicted mice following 8 and 12 weeks of treatment. Immunohistochemical examination demonstrated that there was an increased abnormal distribution of ZO-1 in the bladders of ketamine-treated mice compared with control mice. TEM analysis demonstrated that the surface of bladder urothelium became flattened, the tight junctions between umbrella cells became thinner and the endothelial cells exhibited cell body shrinkage, chromatin condensation and layer denudation in mice treated with ketamine. The present study indicated that the structural and functional changes to the bladder epithelial barrier caused by long-term use of ketamine may be key mechanisms in the development of KIC.
\end{abstract}

Correspondence to: Dr Tianpeng Wu, Department of Urology, Renmin Hospital of Wuhan University, 238 Jiefang Road, Wuhan, Hubei 430060, P.R. China

E-mail: wutpeng@163.com

Key words: ketamine-induced cystitis, permeability barrier, hematoxylin and eosin staining, transmission electron microscope, ultrastructure

\section{Introduction}

Ketamine, which is an $\mathrm{N}$-methyl D-aspartate receptor antagonist, is commonly used as an anesthetic and analgesic agent in human and veterinary procedures due to its low price and its high availability (1); however, research has demonstrated that long-term ketamine abuse may produce adverse symptoms of cystitis, including suprapubic pain, urge incontinence, hematuria and fluctuating lower urinary tract symptoms (LUTS) (2,3), and consequently result in the development of ketamine-induced cystitis (KIC) (4,5). Furthermore, although $\mathrm{KIC}$ has been demonstrated to be induced in abusers using high doses of ketamine $(6,7)$, it has also been observed in prescription users with much lower prescription doses of ketamine (8).

Numerous studies have confirmed that ketamine or its metabolites affect the urothelium of the bladder. Ketamine or its metabolites directly interact with the urothelium and result in defected bladder epithelial barrier, thus inducing the development of $\operatorname{KIC}(9,10)$. Increasing evidence indicates that increased permeability of the urothelial barrier may result in clinical symptoms, such as pain and urgency (11). A study by Jhang et al (12) demonstrated that urothelial barrier dysfunction might be the possible pathophysiology of KIC. Additionally, increased permeability of the urothelial barrier results in bladder epithelial alterations, such as denudation and thinning, which are frequently observed in KIC patients $(4,7)$. Furthermore, decreased expression of E-cadherin and increased urothelial cell apoptosis are observed in KIC tissues, which are considered to be associated with the clinical symptoms of KIC (13). Ketamine is able to promote KIC development via the downregulation of E-cadherin expression in epithelial cells (14). It is therefore possible to speculate that the dysfunction of the bladder epithelial barrier may be responsible for the development of KIC; however, the ultrastructural changes of the bladder epithelial barrier in the pathogenesis of this condition remain unclear.

In the present study, a model of long-term ketamine abuse was created by injecting mice daily with ketamine to investigate the microscopic changes to the epithelial barrier and its surrounding structures. The present study aimed to investigate the changes in the bladder epithelial barrier associated with $\mathrm{KIC}$. The present findings may provide a theoretical basis to elucidate the potential mechanisms of KIC. 


\section{Materials and methods}

Ethics statement. All experimental protocols were approved by the Animal Ethics Committee of Wuhan University Medical Centre (Wuhan, China).

Animals and ketamine administration. A total of 608 -week-old female C57BL/6 mice (weight, 19.08 $\pm 1.29 \mathrm{~g}$ ) were obtained from Renming Hospital of Wuhan University Laboratory Animal Center (Wuhan, China). Mice were maintained under a 12-h light/dark cycle at a constant temperature $\left(21-22^{\circ} \mathrm{C}\right)$ and humidity (50\%). Mice had free access to food and tap water prior to the experiments. Mice were randomly allocated into two groups, control group and ketamine treatment group, and the mice in each group were subsequently subdivided into three subgroups (4, 8 and 12 week groups; $\mathrm{n}=10$ mice/subgroup). Mice received daily intraperitoneal injections of saline (control group) or $100 \mathrm{mg} / \mathrm{kg}$ ketamine (Gutian Fuxing Pharmaceutical Co., Ltd., Gutian, China) (ketamine treatment group) to model the effects of repeated ketamine abuse, which was previously described in a study by Meng et al (15).

Micturition behavior. Micturition frequency was determined as previously described in a study by Gu et al (10). In brief, short-term micturition frequency of freely moving mice was observed at the end of 4,8 and 12 weeks of treatment. At the end of the 4,8 and 12 weeks, respectively, mice were placed in five separate square lattices containing a gridded filter paper pad, with each small grid containing a mouse. Filter paper was impregnated with saturated copper sulfate solution $\left(\mathrm{CuSO}_{4} 5 \mathrm{H}_{2} \mathrm{O}\right)$ and dehydrated at $200^{\circ} \mathrm{C}$ for $1 \mathrm{~h}$ prior to use. When urine fell onto this filter paper, the anhydrous $\mathrm{CuSO}_{4}$ was rehydrated and turned blue. Subsequent to $2 \mathrm{~h}$, the numbers of urine spots $>0.2 \mathrm{~cm}$ in diameter were counted and recorded by five people independently with ultraviolet illumination.

Histopathological and immunohistochemical analysis. Mice were sacrificed with an intraperitoneal injection of sodium pentobarbital (100 mg/kg, Sigma-Aldrich; Merck KGaA, Germany) and bladders were excised for analyses. For histopathlogical analysis, half of the bladder tissues were fixed in $4 \%$ phosphate-buffered paraformaldehyde overnight at room temperature, dehydrated in serial ethanol concentrations, cleared in xylene and embedded into paraffin wax. Serial paraffin sections of $5 \mu \mathrm{m}$ in thickness were created, stained with haematoxylin and eosin and subsequently examined under a light microscope (Nikon Corp., Tokyo, Japan).

For immunohistochemical analysis, tissue sections were dewaxed and rehydrated with graded xylene and serial ethanol concentrations. Subsequently, immunohistochemical labeling for zonula occludens-1 (ZO-1) was performed. Following the blocking of nonspecific antibody activity using Tris-buffered saline (Sigma-Aldrich; Merck KGaA) containing 1\% bovine serum albumin and $10 \%$ fetal calf serum at $37^{\circ} \mathrm{C}$ for $2 \mathrm{~h}$, the tissue sections were incubated with primary antibody anti-ZO-1 (BSA-1543, 1:100 dilution; BioGenex, San Ramon, CA, USA) overnight at $4^{\circ} \mathrm{C}$. Subsequently, hydrogen peroxide was used to eliminate the endogenous peroxidase activity at $37^{\circ} \mathrm{C}$ for $10 \mathrm{~min}$. The distribution of ZO-1 marker was scored into two levels by two independent histologists: Normal and abnormal. If normal,

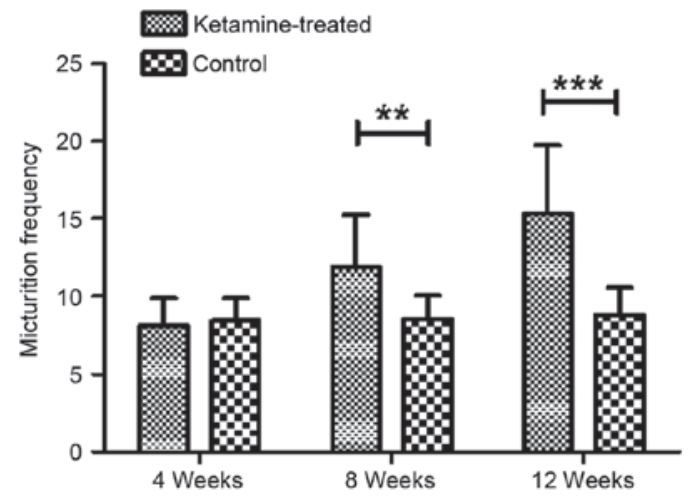

Figure 1. Micturition frequency of freely moving mice measured in a $2-\mathrm{h}$ time period. Data are presented as the mean \pm standard error of the mean. ${ }^{* * *} \mathrm{P}<0.05$ and ${ }^{* * * *} \mathrm{P}<0.01$

the ZO-1 marker was distributed throughout the urothelium and more intensely under the umbrella cell layer with minimal expression in the cytoplasm. If abnormal, the distribution of the ZO-1 marker was patchy, absent, or expressed in the cytoplasm and not localized on cell surfaces.

Ultrastructure of bladder samples. For ultrastructure analysis, half of the bladder tissue samples from each mouse were fixed in $2.5 \%$ glutaraldehyde buffered in $0.1 \mathrm{M}$ phosphate buffer, post-fixed in buffered $1 \%$ osmium tetroxide, dehydrated using ascending grades of ethanol and dry acetone, embedded in epoxy resin, and finally left in a resin-polymerizing oven overnight at $65^{\circ} \mathrm{C}$. The protocol for ultrastructure analysis was in accordance with the method published in the study by Jeong et al (16) with some modifications. Ultrathin sections of $70 \mathrm{~nm}$ in thickness were created, mounted on 200-mesh hexagonal copper grids and stained with lead citrate. The ultrastructural urothelium of the bladder samples was observed using a Hitachi H-600 transmission electron microscope (TEM; Hitachi, Ltd., Tokyo, Japan).

Statistical analysis. Data are expressed as the mean \pm standard error of the mean. Statistical analyses were performed using Prism v.5.0 software (GraphPad Software, Inc., La Jolla, CA, USA). Independent-samples t-tests were used to detect significant differences in micturition frequency between two groups. Fisher's exact test was used to measure any significant differences in $\mathrm{ZO}-1$ expression. $\mathrm{P}<0.05$ was considered to indicate a statistically significant difference.

\section{Results}

Micturition behavior. As exhibited in Fig. 1, the micturition frequency in the ketamine-treated and control groups were determined as $8.05+1.799$ and $8.36+1.492$ following 4 weeks of treatment, and there was no significant difference in micturition frequency between the two groups at this time point $(\mathrm{P}>0.05)$. However, following 8 weeks of treatment, the micturition frequency in the ketamine-treated group was determined as $11.90+3.348$ and was significantly increased compared with that of the control group $(8.50+1.581 ; \mathrm{P}<0.01)$. Similar results were obtained for the micturition frequency in the ketamine-treated group $(15.30+4.423)$ following 12 weeks 
A

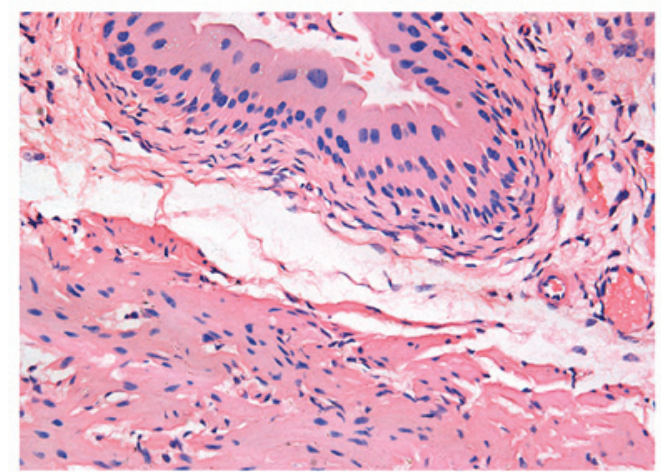

C

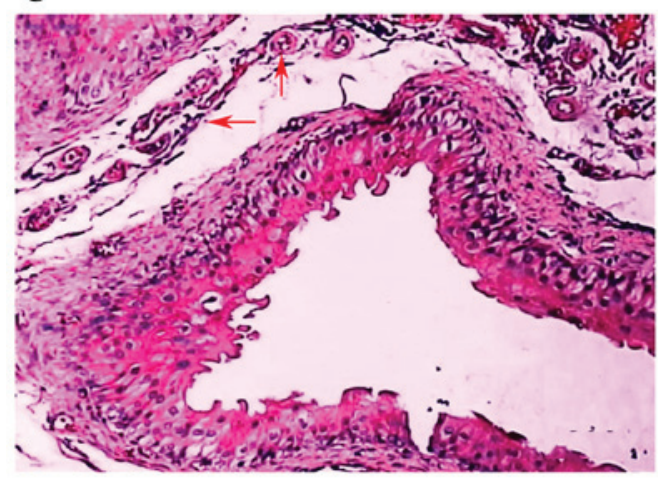

B

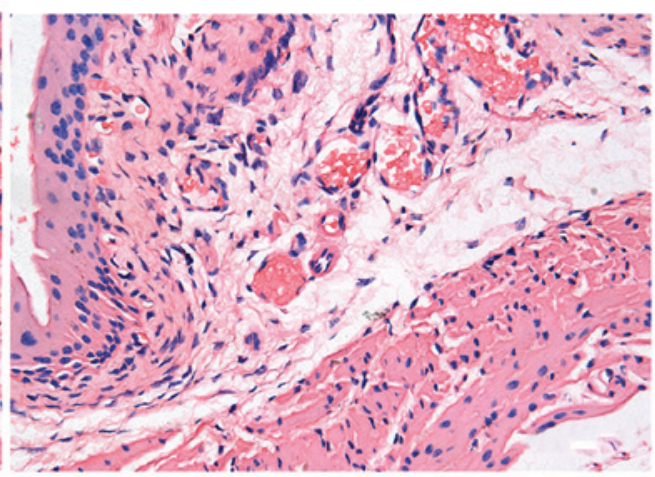

D

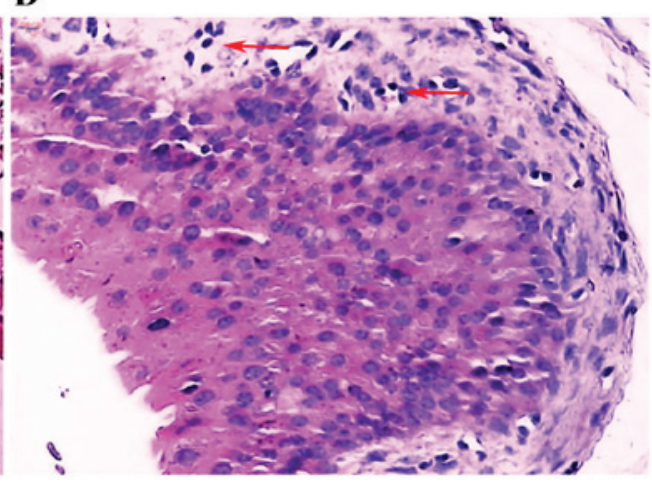

Figure 2. Haematoxylin and eosin staining of midsagittal sections of murine bladders. Bladder sections from (A) the control group (magnification, $\mathrm{x} 400$ ), (B) ketamine-treated mice following 4 weeks of treatment (magnification, $\mathrm{x} 400$ ), (C) ketamine-treated mice following 8 weeks of treatment (magnification, $\mathrm{x} 200)$ and (D) ketamine-treated mice following 12 weeks of treatment (magnification, $\mathrm{x} 400)$.

A

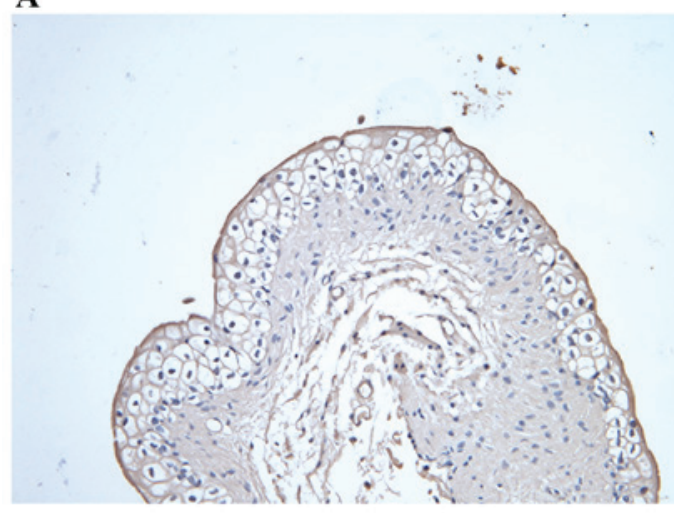

B

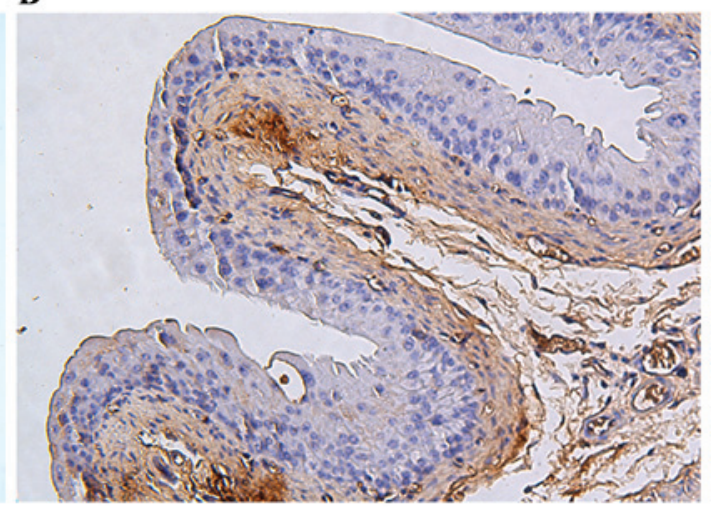

Figure 3. Representative immunohistochemical images of ZO-1 protein. For immunohistochemical analysis, tissue sections were incubated with primary antibody anti-ZO-1 overnight at $4^{\circ} \mathrm{C}$. (A) In control mice, ZO-1 was localized to superficial umbrella cell layer at the interendothelial junctions in most samples. (B) In the ketamine group, ZO-1 located in the cytoplasm and not organized into tight junction structures, or absent. Magnification, x200; scale bar, $100 \mu \mathrm{m}$. ZO-1, zonula occludens-1.

of treatment, and this was significantly higher than that of the control group $(8.50+1.581 ; \mathrm{P}=0.001)$.

Bladder pathology and immunohistochemistry. The urinary bladders of the ketamine-treated mice displayed some pathology differences when compared with the controls. When compared with the control group (Fig. 2A), there was no significant inflammatory cell infiltration and arterial dilatation following 4 weeks of ketamine treatment (Fig. 2B); however, arterial dilatation and congestion were observed under the submucosal epithelium of the urinary bladder following
8 weeks of ketamine treatment (indicated by arrows; Fig. 2C). In addition to the above symptoms, inflammatory cells, predominantly lymphocytes and occasionally macrophages, had infiltrated into the submucosal epithelium of the urinary bladders of mice in the ketamine-treated group after 12 weeks of treatment (indicated by arrows; Fig. 2D).

ZO-1 was localized to the superficial umbrella cell layer at the apicolateral junction in the majority of control group samples (Fig. 3A); however, in the ketamine treatment groups, bladders exhibited a heterogeneous staining distribution, indicating that $\mathrm{ZO}-1$ was distributed in the cytoplasm and was 
Table I. Distribution of ZO-1 protein in each group.

\begin{tabular}{lcccr}
\hline Treatment duration (weeks) & ZO-1 distribution & Ketamine (\%) & Control (\%) & P-value $^{\text {a }}$ \\
\hline 4 & & & & 0.003 \\
& Normal & $3(30)$ & $10(100)$ & 0.022 \\
Abnormal & $7(70)$ & & $0(0)$ & 0.001 \\
& Normal & $3(30)$ & $1(10)$ & $9(90)$ \\
Abnormal & & $7(70)$ & $1(10)$ & \\
\hline
\end{tabular}

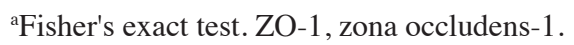

not absent or organized into tight junction structures (Fig. 3B). Additionally, the number of samples exhibiting abnormal ZO-1 distribution in the ketamine-treated group was increased compared with control group, with abnormal ZO-1 distribution in 70 vs. $0 \%(\mathrm{P}=0.003)$ following 4 weeks, 70 vs. $10 \%$ $(\mathrm{P}=0.022)$ following 8 weeks and 90 vs. $10 \%(\mathrm{P}=0.001)$ following 12 weeks of treatment in the ketamine-treated and control groups, respectively (Table I).

Ultrastructure of bladder samples. Morphological ultrastructural alterations to the uroepithelium and lamina propria of bladder cells were determined using TEM. As demonstrated in Fig. 4A-D, the surface of the umbrella cells in the control group were suffused with raised microplicae (indicated by arrows in Fig. 4A and B) and the cytoplasm of these cells contained multiple subapical vesicles (indicated with asterisks in Fig. 4B). Marked changes in bladder morphology were observed in the ketamine-treated group compared with the control group. The surface of the umbrella cells appeared more flattened following 4 (Fig. 4E-H) and 8 weeks (Fig. 4I-L), and even diminished following 12 weeks (Fig. 4M-P), of treatment in the ketamine-treated group compared with the control group. Thin tight junction complexes were frequently observed between the umbrella cells following 4 weeks of treatment in the ketamine-treated group (Fig. 4G); however, these distinct junction complexes were not observed following 8 and 12 weeks of ketamine treatment (Fig. $4 \mathrm{~K}$ and O). In addition, the vascular endothelial cells exhibited cell body shrinkage, increased cytoplasm density and chromatin condensation following 8 weeks (Fig. 4L) and layer denudation following 12 weeks (Fig. 4P) of ketamine treatment.

\section{Discussion}

In the present study, a 12-week murine model of ketamine abuse was created in order to characterize ultrastructural changes of the bladder epithelial barrier and its surrounding structures as a result of long-term ketamine abuse, thus elucidating the pathogenesis of KIC. Following 8 and 12 weeks of ketamine treatment, the frequency of micturitions in ketamine-treated mice was significantly increased compared with that of the controls. Additionally, histopathological analyses of the ketamine-treated bladders exhibited suburothelial congestion and mononuclear cell infiltration, and immunohistochemistry results revealed an increased abnormal distribution of ZO-1 marker in the bladders of ketamine-treated mice compared with controls. TEM analysis revealed that the surface of umbrella cells appeared more flattened, tight junctions between umbrella cells became thinner and the endothelial cells exhibited cell body shrinkage, chromatin condensation and layer denudation in ketamine-treated mice compared with the controls. These results indicate that ketamine may alter the structure and function of the bladder epithelial barrier, resulting in urinary tract damage and the development of KIC.

It has previously been reported that the long-term use of illicit ketamine is strongly associated with KIC development (17). Research has demonstrated that long-term use of illicit ketamine is able to produce adverse effects in the urinary tract and induce increased micturition frequency in a young Malay woman (18). Furthermore, a study by Meng et al (15) demonstrated that micturition frequency increased and bladder capacity decreased following 8 weeks of daily ketamine treatment in mice. In the present study, the micturition frequency was increased after 8 and 12 weeks of ketamine treatment in mice compared with saline-injected controls, suggesting that long-term ketamine use may induce urinary tract damage in mice. In addition, a previous study has demonstrated the characteristics of long-term ketamine use in humans with a history of LUTS (19). Bladder epithelium has been demonstrated to have an important function in providing an impermeable barrier to urinary solutes, including ammonia, potassium, urea and creatinine (20). The damaged barrier may result in the leakage of urine components into the urothelium layers, resulting in irritation, inflammation and symptoms of LUTS (21). In the present study, histopathological analyses of the ketamine-treated bladders exhibited suburothelial congestion and mononuclear cell infiltration, which were consistent with previous findings after chronic ketamine treatment in mice $(15,22)$. The results of the present study suggest that long-term ketamine use may induce bladder dysfunction and subsequently promote KIC development. Although the results suggest that urothelial barrier defects occur in KIC, 

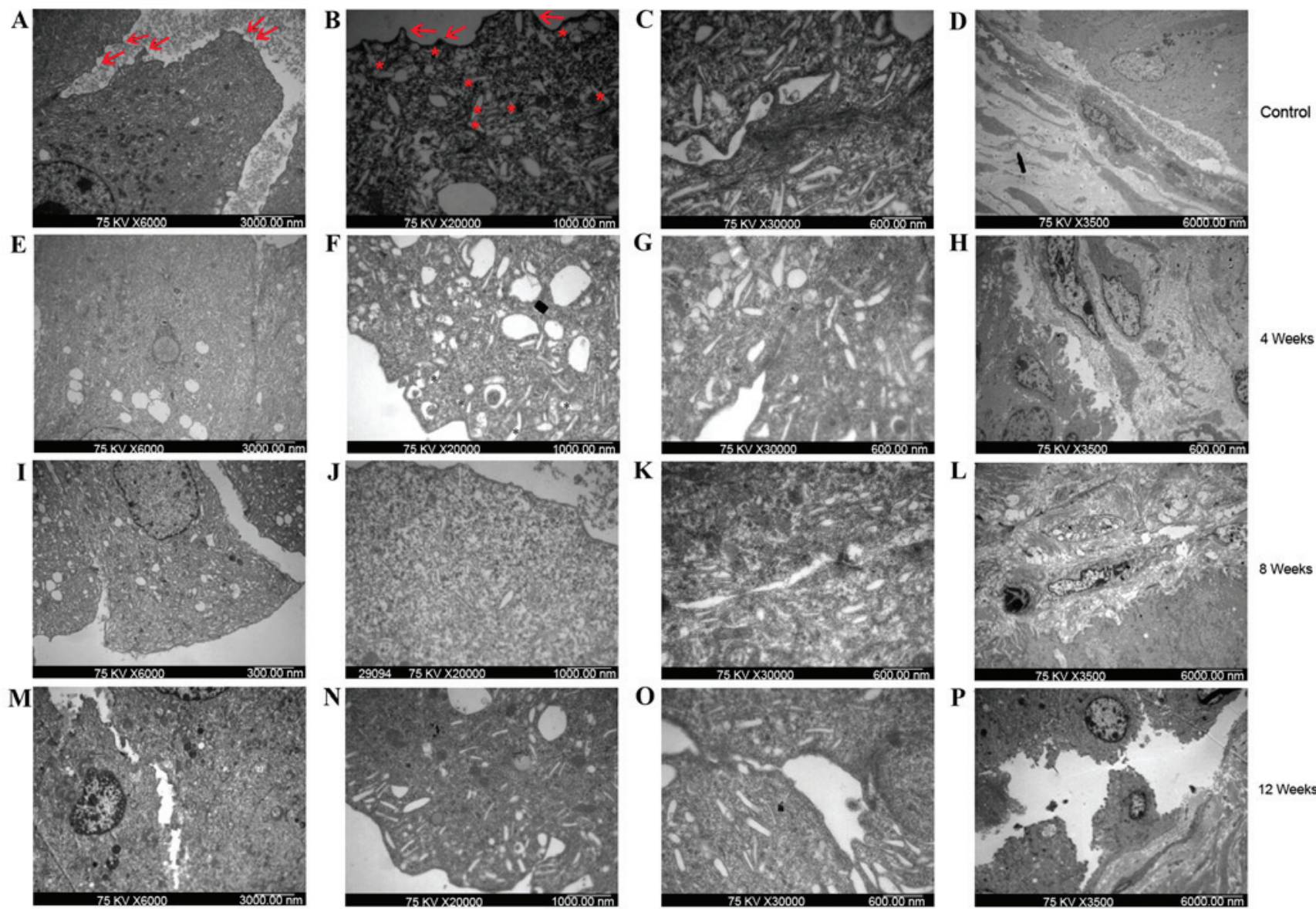

Figure 4. Bladder uroepithelium and lamina propria from control and ketamine-treated mice detected using transmission electron microscopy. Bladder samples from (A-D) control mice and ketamine-treated mice following (E-H) 4, (I-L) 8 and (M-P) 12 weeks of treatment. (A, E, I and M) Cell-cell connections (magnification, $\mathrm{x} 6,000$; scale bar, 3,000 nm). The gap between cells in the ketamine-treated groups $(\mathrm{E}, \mathrm{I}$ and $\mathrm{M})$ was wider than that observed in the control group (A). (B, F, J and N) Apical membrane of umbrella cells (magnification, x20,000; scale bar, 1,000 nm). The control group (B) exhibited a normal appearance, with numerous subapical vesicles (asterisks) and raised microplicaes (arrows). Rare subapical vesicles and raised microplicaes were observed in the ketamine treatment groups (F, J and L). (C, G, K and O) Junctional complexes (magnification, x30,000; scale bar, $600 \mathrm{~nm}$ ). Control group (C) exhibited distinct tight junctional complexes, whereas the ketamine treatment groups ( $\mathrm{G}, \mathrm{K}$ and $\mathrm{O}$ ) exhibited broken junctional complexes. Cells from the bladders of mice treated with ketamine for 4 weeks lost their cytoplasmic density. (D, H, L and P) Bladder lamina propria (magnification, x3,500; scale bar, 6,000 nm). The control group and 4-week ketamine treatment group exhibited normal appearance of lamina propria. Vascular endothelial cells of the 8-week ketamine treatment group exhibited cell body shrinkage, cytoplasm density increase and chromatin condensation. The 12-week ketamine treatment group exhibited discontinuity in the umbrella cell layer and denuded epithelium.

it cannot be explained why ketamine and its metabolites are able to damage the urothelial cells and, therefore, this may be a limitation of the present study. Notably, a previous study demonstrated that a significant number of urothelial atypia mimicking carcinoma were observed in situ (2). In the present study, bladders of ketamine-treated mice exhibited squamous cell metaplasia following 12 weeks of treatment, leading to the hypothesis that long-term ketamine abuse may eventually contribute to the development of bladder cancer; however, whether ketamine abuse would eventually result in the development of bladder cancer is unknown and long-term follow-up studies are required.

Tight junctions between urothelial cells are essential components of the permeability barrier, and tight junction proteins, including ZO-1, are believed to have important roles in maintaining epithelial barrier function and the impermeability of the bladder urothelium $(23,24)$. Furthermore, tight junctions are able to block transepithelial ion flux, which is conducive to maintaining the transepithelial resistance of the epithelium (25). Accompanied by the disruption of tight junctions, surface epithelial cells exhibit raised microplicae, which are believed to fuse with the apical plasma membrane in response to changes in basolateral osmolality or stretch (26). ZO-1 forms tight junctions, which contributes to the impermeability of the bladder urothelium $(26,27)$. Expression of ZO-1 has been demonstrated to decrease following in vitro exposure to antiproliferative factor, which has been proposed to be a sensitive and specific biomarker for interstitial cystitis (IC) $(28,29)$. In the present study, thin tight junction complexes and separation of cell-cell contacts were observed in ketamine-treated mice using TEM. As well as the observation of ultrastructural changes, abnormal expression of ZO-1 proteins was observed following 8 and 12 weeks of ketamine treatment, which was also observed in bladder biopsy specimens from patients with IC (30). Therefore, the results of the present study suggest that long-term ketamine use may alter the permeability of the bladder epithelial barrier in the pathogenesis of KIC; however, particular limitations of this study should be acknowledged. Firstly, the present study was descriptive in nature and provided little mechanistic insight. Secondly, the results of the present 
study imply that ketamine treatment may lead to alterations to the permeability of bladder epithelial barrier; however, functional assessment of permeability was not conducted. Thirdly, the distribution of ZO-1 was described and not quantified, which reduces the conviction of the present study to a certain extent. Therefore, more experimental validations, such as functional assessment of permeability and qualitative and/or quantitative histopathological evaluation of ketamine-induced bladder lesions, are required.

In conclusion, the present study indicated that the structural and functional alterations to the bladder epithelial barrier as a result of the long-term use of ketamine may be key mechanisms in the development of KIC.

\section{Acknowledgements}

The present study was financially supported by grants from the Hubei Natural Science Foundation (grant no. 2013CKB023).

\section{References}

1. Sha O, Hao Y, Cho EY and Zhou L: Clinical applications and side effects of ketamine. Ketamine Use and Abuse: 13-36, 2015.

2. Oxley JD, Cottrell AM, Adams S and Gillatt D: Ketamine cystitis as a mimic of carcinoma in situ. Histopathology 55: 705-708, 2009.

3. Mason K, Cottrell AM, Corrigan AG, Gillatt DA and Mitchelmore AE: Ketamine-associated lower urinary tract destruction: A new radiological challenge. Clin Radiol 65: 795-800, 2010.

4. Chu PS, Kwok SC,Lam KM, Chu TY, Chan SW, Man CW, Ma WK, Chui KL, Yiu MK, Chan YC, et al: Street ketamine'-associated bladder dysfunction: A report of ten cases. Hong Kong Med J 13: 311-313, 2007.

5. Middela S and Pearce I: Ketamine-induced vesicopathy: A literature review. Int J Clin Pract 65: 27-30, 2011.

6. Chu PS, Ma WK, Wong SC, Chu RW, Cheng CH, Wong S, Tse JM, Lau FL, Yiu MK and Man CW: The destruction of the lower urinary tract by ketamine abuse: A new syndrome? BJU Int 102: 1616-1622, 2008

7. Tsai TH, Cha TL, Lin CM, Tsao CW, Tang SH, Chuang FP, Wu ST, Sun GH, Yu DS and Chang SY: Ketamine-associated bladder dysfunction. Int J Urol 16: 826-829, 2009.

8. Grégoire MC, MacLellan DL and Finley GA: A pediatric case of ketamine-associated cystitis (Letter-to-the-Editor RE: Shahani R, Streutker C, Dickson B, Stewart RJ: Ketamine-associated ulcerative cystitis: A new clinical entity. Urology 69: 810-812, 2007). Urology 71: 1232-1233, 2008.

9. Parkin MC, Turfus SC, Smith NW, Halket JM, Braithwaite RA, Elliott SP, Osselton MD, Cowan DA and Kicman AT: Detection of ketamine and its metabolites in urine by ultra high pressure liquid chromatography-tandem mass spectrometry. J Chromatogr B Analyt Technol Biomed Life Sci 876: 137-142, 2008.

10. Gu D, Huang J, Yin Y, Shan Z, Zheng S and Wu P: Long-term ketamine abuse induces cystitis in rats by impairing the bladder epithelial barrier. Mol Biol Rep 41: 7313-7322, 2014.

11. Parsons CL: The role of a leaky epithelium and potassium in the generation of bladder symptoms in interstitial cystitis/overactive bladder, urethral syndrome, prostatitis and gynaecological chronic pelvic pain. BJU Int 107: 370-375, 2011.
12. Jhang JF, Hsu YH and Kuo HC: Possible pathophysiology of ketamine-related cystitis and associated treatment strategies. Int J Urol 22: 816-825, 2015.

13. Lee CL, Jiang YH and Kuo HC: Increased apoptosis and suburothelial inflammation in patients with ketamine-related cystitis: A comparison with non-ulcerative interstitial cystitis and controls. BJU Int 112: 1156-1162, 2013.

14. Li M, Yang K, Wang X, Xu X, Zhu L and Wang H: Mast cells infiltration and decreased E-cadherin expression in ketamine-induced cystitis. Toxicol Rep 2: 205-209, 2015.

15. Meng E, Chang HY, Chang SY, Sun GH, Yu DS and Cha TL: Involvement of purinergic neurotransmission in ketamine induced bladder dysfunction. J Urol 186: 1134-1141, 2011.

16. Jeong SH, Kim JH, Yi SM, Lee JP, Kim JH, Sohn KH, Park KL, Kim MK and Son SW: Assessment of penetration of quantum dots through in vitro and in vivo human skin using the human skin equivalent model and the tape stripping method. Biochem Biophys Res Commun 394: 612-615, 2010.

17. Morgan CJ and Curran HV; Independent Scientific Committee on Drugs: Ketamine use: A review. Addiction 107: 27-38, 2012.

18. Noorzurani R, Vicknasingam B and Narayanan S: Illicit ketamine induced frequency of micturition in a young Malay woman. Drug Alcohol Rev 29: 334-336, 2010.

19. Chao JY and Shai HA: Duloxetine treatment of long-term ketamine abuse-related lower urinary tract symptoms: A case report. Gen Hosp Psychiatry 32: 647.e5-e6, 2010.

20. Madersbacher H, van Ophoven A and van Kerrebroeck PE: GAG layer replenishment therapy for chronic forms of cystitis with intravesical glycosaminoglycans-a review. Neurourol Urodyn 32: 9-18, 2013.

21. Keay SK, Birder LA and Chai TC: Evidence for bladder urothelial pathophysiology in functional bladder disorders. Biomed Res Int 2014: 865463, 2014.

22. Yeung L, Rudd JA, Lam WP, Mak YT and Yew DT: Mice are prone to kidney pathology after prolonged ketamine addiction. Toxicol Lett 191: 275-278, 2009.

23. Gonzalez-Mariscal L, Betanzos A, Nava P and Jaramillo BE: Tight junction proteins. Prog Biophys Mol Biol 81: 1-44, 2003.

24. Matter K and Balda MS: Signalling to and from tight junctions. Nat Rev Mol Cell Biol 4: 225-237, 2003.

25. Wang EC, Lee JM, Johnson JP, Kleyman TR, Bridges R and Apodaca G: Hydrostatic pressure-regulated ion transport in bladder uroepithelium. Am J Physiol Renal Physiol 285: F651-F663, 2003.

26. Lavelle J, Meyers S, Ramage R, Bastacky S, Doty D, Apodaca G and Zeidel ML: Bladder permeability barrier: Recovery from selective injury of surface epithelial cells. Am J Physiol Renal Physiol 283: F242-F253, 2002.

27. Sterle M, Veranic P and Jezernik K: Exogenously added growth factors have no effect on formation of cell junctions and cytoskeleton in urothelial cells in culture. Pflugers Arch 439 (Suppl 3): R143-R144, 2000.

28. Zhang CO, Wang JY, Koch KR and Keay S: Regulation of tight junction proteins and bladder epithelial paracellular permeability by an antiproliferative factor from patients with interstitial cystitis. J Urol 174: 2382-2387, 2005.

29. Keay S: Cell signaling in interstitial cystitis/painful bladder syndrome. Cell Signal 20: 2174-2179, 2008.

30. Slobodov G, Feloney M, Gran C, Kyker KD, Hurst RE and Culkin DJ: Abnormal expression of molecular markers for bladder impermeability and differentiation in the urothelium of patients with interstitial cystitis. J Urol 171: 1554-1558, 2004. 